\title{
Cholesteryl ester transfer protein inhibitors for dyslipidemia: focus on dalcetrapib
}

This article was published in the following Dove Press journal:

Drug Design, Development and Therapy

2I September 2012

Number of times this article has been viewed

\author{
Alyse S Goldberg \\ Robert A Hegele \\ Department of Medicine, Schulich \\ School of Medicine and Dentistry, \\ Western University, London, Ontario, \\ Canada
}

\begin{abstract}
Among the noteworthy recent stories in the management and prevention of atherosclerotic cardiovascular disease (CVD) is the saga of the development of pharmacological inhibitors of cholesteryl ester transfer protein (CETP). Inhibiting CETP significantly raises plasma concentrations of high-density lipoprotein cholesterol, which has long been considered a marker of reduced CVD risk. However, the first CETP inhibitor, torcetrapib, showed a surprising increase in CVD events, despite a dramatic increase in high-density lipoprotein cholesterol levels. This paradox was explained by putative off-target effects not related to CETP inhibition that were specific to torcetrapib. Subsequently, three newer CETP inhibitors, namely dalcetrapib, anacetrapib, and evacetrapib, were at various phases of clinical development in 2012. Each of these had encouraging biochemical efficacy and safety profiles. Dalcetrapib even had human arterial imaging results that tended to look favorable. However, the dalcetrapib development program was recently terminated, presumably because interim analysis of a large CVD outcome trial indicated no benefit. These events raise important questions regarding the validity of the mechanism of CETP inhibition and the broader issue of whether pharmacological raising of high-density lipoprotein cholesterol itself is a useful strategy for CVD risk reduction.
\end{abstract}

Keywords: dalcetrapib, CETP inhbitor, HDL, cardiovascular disease

\section{Background}

Despite the success of statin therapy in targeting low-density lipoprotein (LDL) cholesterol to reduce cardiovascular disease (CVD) event rates by $30 \%-40 \%$, CVD risk has not been abolished with statin treatment. ${ }^{1}$ Low serum concentration of highdensity lipoprotein (HDL) cholesterol $(<0.9 \mathrm{mmol} / \mathrm{L})$ is considered by many to be the strongest lipid risk factor for CVD and coronary artery disease. ${ }^{2}$ Epidemiological studies consistently suggest that a $0.26 \mathrm{mmol} / \mathrm{L}$ increase in HDL cholesterol level is associated with $2 \%-3 \%$ decrease in CVD events. ${ }^{3}$ This possible role of HDL cholesterol in explaining the residual risk among patients who suffer CVD events despite achieving target LDL cholesterol levels has led to interest in targeting biochemical pathways to increase HDL cholesterol. One such mechanism is inhibition of cholesteryl ester transfer protein (CETP). The purpose of this paper is to address the role of CETPtargeted therapy for reducing CVD risk, with the focus on the case of dalcetrapib, the development of which was recently terminated.

\section{HDL particles}

The major structural proteins that form the scaffold for HDL are apolipoprotein (apo) A-I and apo A-II. ${ }^{4}$ The heterogeneous subpopulations of HDL particles vary in 
shape, density, size, composition, and surface charge, and have been given a variety of designations over the years. One system includes terms such as pre- $\beta, \alpha-1, \alpha-2, \alpha-3$, pre- $\alpha-1$, pre- $\alpha-2$, and pre- $\alpha-3$ HDL, but these have no clinical correlates, because the subfractions are identified using a research-intensive method that is unavailable in clinical laboratories. $^{5}$

HDL begins its life cycle as a small, flat, lipid-poor discoidal particle, termed pre- $\beta$ HDL, which is secreted directly from the liver or intestine or which can be assembled in the plasma (Figure 1). At the surface of peripheral cells, cholesteryl ester (CE) and free cholesterol are transported from the cell interior to the small nascent HDL particles by ATP-binding cassette subfamily members G1 and A1, respectively. The action of lecithin-cholesterol acyltransferase on these lipid substrates results in enlargement and maturation of spherical HDL particles, which journey back to the liver and constitute the predominant form of circulating HDL. Density-based ultracentrifugation divides these larger HDLs into $\mathrm{HDL}_{2}$ and $\mathrm{HDL}_{3}$ subfractions, with $\mathrm{HDL}_{2}$ being larger and less dense than $\mathrm{HDL}_{3} \cdot{ }^{6}$

Two broad types of atheroprotective mechanisms have been attributed to HDL: (1) its role in reverse cholesterol transport (RCT) and (2) its multiple beneficial "pleiotropic effects," including protecting against oxidative stress and inflammation and promoting endothelial repair and function. ${ }^{7,8}$

\section{HDL functionality}

When HDL cholesterol is measured clinically, the reported value does not directly reflect the number or type of particles, but rather it indicates the amount of cholesterol that is contained within the entire HDL lipoprotein fraction. ${ }^{4}$ There is no information provided on how the cholesterol is distributed among the various HDL subfractions, nor is there any implication for the HDL function, for instance, its role in RCT or its multiple pleiotropic effects.

It appears that not all HDLs are equally functional and that distinct subpopulations have different cardioprotective properties. ${ }^{6}$ For instance, among the HDL particles distinguished by ultracentrifugation, $\mathrm{HDL}_{2}$ - the larger, less dense HDL - may be particularly important at some stages of RCT, ${ }^{9}$ and in some studies, its concentration is the best negative predictor of CVD events. ${ }^{10}$ These particles are also inversely correlated with atherosclerosis, hypertriglyceridemia, and CVD. ${ }^{11,12}$ In contrast, the smaller, denser $\mathrm{HDL}_{3}$ subfraction may protect LDL from oxidative stress, while very small particles, such as pre- $\beta$ HDL, are more functionally active in RCT. ${ }^{13}$ Fractions that can be distinguished on gradient gels also appear to have functional differences. For instance,

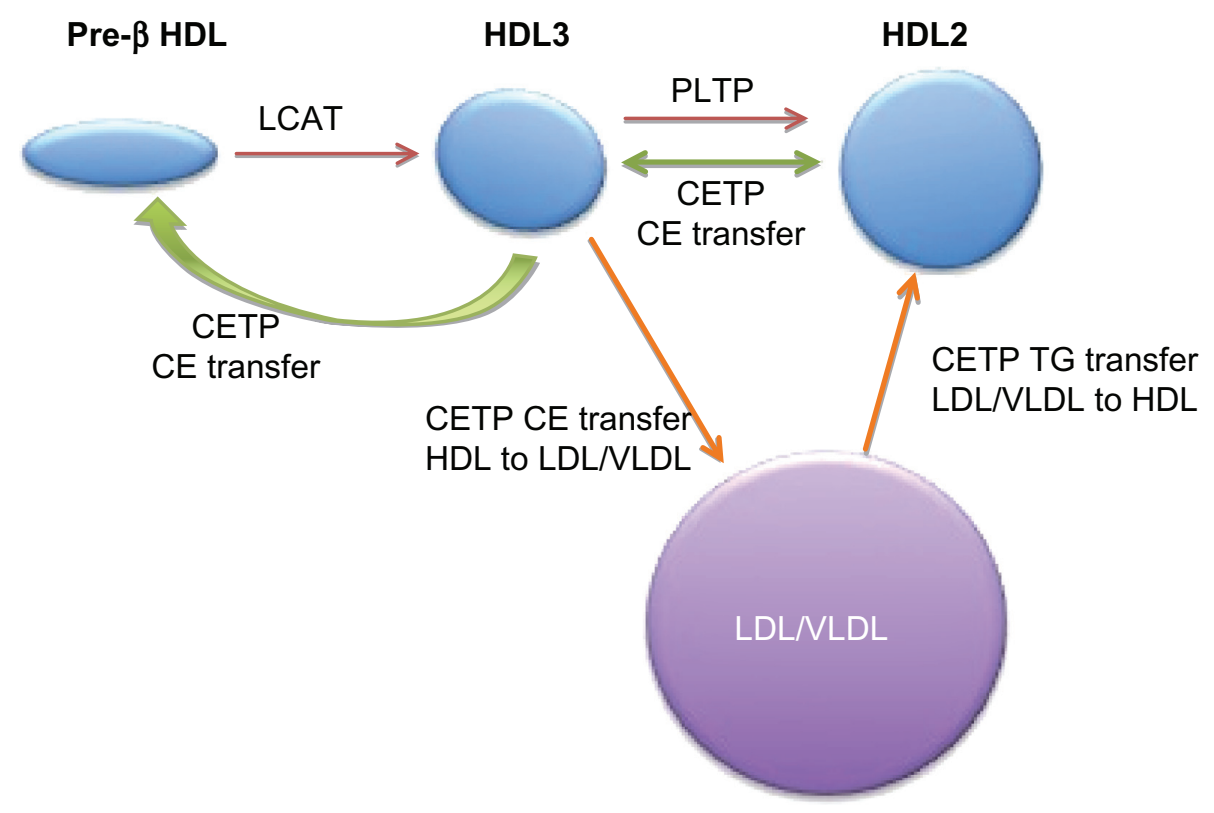

Figure I HDL lifecycle and CETP function.

Notes: Pre- $\beta$ HDL (lipid-poor apo A-I HDL particles) is converted to spherical $\mathrm{HDL}_{3}$ by lecithin-cholesterol acyltransferase (LCAT). HDL $\mathrm{L}_{3}$ is converted to $\mathrm{HDL}_{2}$ by fusion of small HDL particles activated by phospholipid transfer protein (PLTP). The modulator role of CETP transfers cholesteryl esters (CE) between HDL particles to create both larger $\mathrm{HDL}_{2}$ and smaller pre- $\beta \mathrm{HDL}$ particles (green arrows), which may be an antiatherogenic process. CETP also mediates heterotropic transfer of triglyceride between LDL/VLDL and HDL (orange arrows), as discussed in the text.

Abbreviations: CETP, cholesteryl ester transfer protein; HDL, high-density lipoprotein; LDL, low density lipoprotein; VLDL, very low density lipoprotein. 
lower $\alpha-1$ and pre- $\alpha-1$ but higher $\alpha-3$ HDL subfractions are evident in patients with CVD, ${ }^{5}$ and low $\alpha-1$ and $\alpha-2$ levels predict CHD. ${ }^{14,15}$ However, these differential functions attributed to specific subclasses of HDL are largely in vitro phenomena, as yet with little direct correlation with CVD surrogate endpoints. ${ }^{16}$

\section{Multiple means of raising HDL}

Current modes of increasing HDL include lifestyle changes (such as weight loss, smoking cessation, reducing intake of trans and saturated fats, and increasing exercise) and certain medications - in particular, fibrates and niacin. In addition to raising HDL cholesterol levels, all of these modalities have the added benefit of decreasing plasma triglyceride (TG) levels, ${ }^{4,17}$ a biochemical effect that has not been demonstrated consistently with CETP inhibition. Moderate alcohol intake also can raise HDL cholesterol, but often increases TG levels concurrently; because alcohol is an addictive substance, it is not typically advised as a "lifestyle" means of raising HDL cholesterol.

Among existing pharmacotherapies, fibrates have a relatively small effect on HDL cholesterol levels (an approximately $10 \%$ increase), while the impact of niacin is greater (an approximately 25\% increase, or more at the highest doses). Niacin's HDL-raising effect has been known for at least 50 years, and this has always been considered beneficial. However, niacin's role in preventing CVD by increasing HDL cholesterol was recently called into question after the premature stopping of the Atherothrombosis Intervention in Metabolic Syndrome with Low HDL Cholesterol/High Triglyceride and Impact on Global Health Outcomes (AIM$\mathrm{HIGH}$ ) trial because of its futility. ${ }^{18}$ The possible reasons for this disappointing outcome include a higher-than-expected HDL cholesterol increase in the control group and very low levels of LDL cholesterol associated with longstanding pretreatment with statin drugs in both treatment groups. This might have left minimal unstable lipid-rich plaques remaining that could benefit from HDL raising. Also, the sample size, in retrospect, was relatively small (approximately 3400 patients), and the study was likely statistically too underpowered to detect hard significant differences in CVD outcomes. Mechanistically, niacin has been shown to be associated with increased $\alpha-1$ HDL levels, which should be of benefit. ${ }^{19}$ Optimism thus persists for the very large Heart Protection Study 2 (HPS2-THRIVE), scheduled to report in 2013, which has enrolled approximately 25,000 patients and should provide the definitive word on the clinical efficacy of extended-release niacin in CVD risk reduction. ${ }^{20}$

\section{CETP}

Circulating CETP is produced by the liver and is bound to small HDL particles and nascent discoid-shaped HDL (Figure 1). ${ }^{21}$ In plasma, CETP facilitates the exchange of $\mathrm{CE}$ from HDL for TG from apo B-containing lipoproteins, which include LDL, intermediate-density lipoprotein, and very low density lipoprotein. This "heterotropic" exchange, or "heterotransfer," results in CE-enriched apo B particles and HDL particles with high TG content. Further, the HDL particles that are derived from CETP activity are small and can be renally excreted, resulting in low HDL cholesterol levels. However, the net result of lowering HDL cholesterol and increasing LDL cholesterol led to the concept that inhibiting CETP would result in a favorable LDL:HDL cholesterol ratio, which in turn would slow atherogenesis and reduce clinical events. ${ }^{4}$ The CETP-related increase in the CE content of atherogenic apo B-containing lipoproteins may not be deleterious in the context of the normal physiological capacity for LDL-particle uptake and catabolism.

\section{CETP modes of action}

CETP may contribute to both HDL atheroprotective mechanisms. For instance, as part of RCT, CETP plays a critical role in transferring to apo B-containing lipoproteins $\mathrm{CE}$ that originated from peripheral tissues, driving uptake and disposal of cholesterol by the liver. ${ }^{4}$ Also, inhibition of CETP blocks this transfer and can lead to an accumulation of HDL particles, which should also be beneficial, since these particles are proposed to have multiple beneficial effects on vascular biology, inflammation, and coagulation.

Ultimately, the goal of CETP inhibition is to increase plasma-HDL cholesterol and thereby reduce CVD risk. Interestingly, reduced CVD risk has been observed less than consistently among patients with naturally occurring CETP mutations, providing a clue that reduced CETP activity is not automatically favorable. ${ }^{22}$ However, CETP also transfers lipids between HDL subspecies through a process called "homotransfer," which remodels HDL and raises levels of certain subspecies, including $\mathrm{HDL}_{2}$ and a smaller pre- $\beta$ HDL from $\mathrm{HDL}_{3 .}{ }^{21} \mathrm{HDL}$ remodeling also permits apo A-I to accept CE from ATP-binding cassette subfamily member $\mathrm{A} 1,{ }^{4}$ a process that has antiatherogenic potential. However, the balance in vivo between these manifold mechanisms and the predicted integrated effects of CETP inhibition are not clear-cut.

The subtleties of HDL structure and function might clarify both the complexity and the sometimes counterintuitive effects of CETP inhibition on clinical outcomes. 
Further, different CETP inhibitors have different effects on heterotransfer of lipids between HDL and apo B-containing lipoproteins and on homotransfer, among other differences in effects. Understanding the intricacies of what differentiates the various CETP inhibitors thus requires a deeper appreciation of HDL structure and function.

\section{Past and present CETP inhibitors for dyslipidemia}

Torcetrapib (Pfizer Inc, New York, NY) was the first CETP inhibitor to progress to Phase III clinical trials. Initially, animal studies were encouraging: they demonstrated that torcetrapib increased HDL cholesterol concentrations approximately three-fold and reduced atherosclerotic plaque by $60 \%{ }^{23}$ In humans, increases in HDL cholesterol of up to $91 \%$ were seen with monotherapy, ${ }^{24}$ and up to $60 \%$ reductions were seen with concurrent use of statins, ${ }^{25}$ sparking great enthusiasm for this therapeutic strategy; this was, however, tempered by early recognition of a modest effect on blood pressure, with increases of 1.3-2.2 mmHg in systolic blood pressure and $0.9-1.1 \mathrm{mmHg}$ in diastolic blood pressure at doses of 60 or $90 \mathrm{mg} /$ day. ${ }^{25}$ Also, approximately $4 \%$ of individuals showed a $>15 \mathrm{mmHg}$ increase in blood pressure, ${ }^{26}$ but most opinion leaders at the time considered this to be of only marginal clinical importance, compared with the lipidmodulating benefits of torcetrapib. ${ }^{27}$

Unfortunately, the mood abruptly changed in 2006 when a pivotal large-scale clinical trial, namely Investigation of Lipid Level Management to Understand Its Impact in Atherosclerotic Events (ILLUMINATE) ${ }^{28}$ was prematurely discontinued after torcetrapib was associated with an increased risk of CVD events and death, despite a $72 \%$ increase in HDL cholesterol and a $25 \%$ decrease in LDL cholesterol levels. The concurrent cardiovascular surrogate outcome trials, including ILLUSTRATE (Investigation of Lipid Level Management Using Coronary Atherosclerosis by CETP Inhibition and HDL Elevation), ${ }^{29}$ RADIANCE-1 (Rating Atherosclerosis Disease Change with a New CETP Inhibitor), ${ }^{30}$ and RADIANCE-2, ${ }^{31}$ all showed no change in coronary or carotid atherosclerosis, and no differences in regression of atherosclerosis in patients who received torcetrapib. ${ }^{29,31}$ Subsequent post hoc analysis did rekindle hope, as some patients from ILLUSTRATE, who were treated with torcetrapib and were within the highest quartile of HDL cholesterol increase, actually showed regression in coronary plaques. ${ }^{32}$

The clinical failure of torcetrapib has subsequently been attributed to "off target" effects on aldosterone and cortisol levels, ${ }^{33,34}$ electrolytes, blood-pressure changes, and prolonga- tion of QT interval on the electrocardiogram. But some still consider that a cleaner drug - one with pure CETP inhibition that is independent of off-target effects - could still be an effective therapeutic approach. ${ }^{17}$ However, the question remains as to whether the mechanism of CETP inhibition is not itself therapeutically beneficial: perhaps CETP inhibition generates large amounts of large HDL particles that are not optimally functional. ${ }^{6}$ Nevertheless, to avoid another failure similar to that of torcetrapib, development of the subsequent CETP inhibitors anacetrapib (Merck and Co, Inc, Whitehouse Station, NJ), evacetrapib (Eli Lilly and Company, Indianapolis, IN), and dalcetrapib (Roche, Basel, Switzerland) included early safety monitoring, with especially close attention paid to changes in blood pressure. ${ }^{19,27}$

\section{Anacetrapib and evacetrapib}

Anacetrapib appears to be the most potent CETP inhibitor; it reduces CETP activity by up to $90 \%$ within 24 hours. ${ }^{35} \mathrm{As}$ a result, it has impressive HDL cholesterol-raising and LDL cholesterol-lowering capabilities both as monotherapy and in combination with a statin. In the DEFINE study, ${ }^{36}$ designed to assess the efficacy and safety of anacetrapib, Cannon et al conducted a multicenter, randomized, double-blind, placebocontrolled trial, and showed that LDL cholesterol was reduced by $36 \%$, that HDL cholesterol was increased by $139 \%$, and that there was no effect on TG in patients with anacetrapib together with a statin. No significant effect on high-sensitivity $\mathrm{C}$-reactive protein was seen with anacetrapib monotherapy. ${ }^{36}$ Meanwhile, dose-dependent decreases in lipoprotein(a) (Lp[a]) of up to $50 \%$ were observed, which is another potential benefit. ${ }^{37}$ Regarding safety, many studies have shown that the deleterious effects of torcetrapib - such as increases in blood pressure, plasma corticosterone, and aldosterone, and increased induction of two steroidogenic enzymes (CYP11B1 and CYP11B2) involved in the last step of cortisol and aldosterone biosynthesis - are not seen with anacetrapib. ${ }^{33,34}$ Overall, to date, anacetrapib has been generally well tolerated clinically, ${ }^{37-40}$ with no evidence of blood-pressure elevations. ${ }^{37,38,41}$

A more recent addition to the CETP-inhibitor family is evacetrapib. Similar to anacetrapib, early-phase clinical trials with evacetrapib were promising, with no effects on blood pressure or aldosterone levels. When administered in the presence of a statin to patients with dyslipidemia, $100 \mathrm{mg}$ evacetrapib inhibited CETP activity by $89 \%$, reduced LDL cholesterol by approximately $12 \%$, and increased HDL cholesterol by approximately $80 \% .{ }^{42}$ Additionally, evacetrapib had no effect on high-sensitivity C-reactive protein, 
blood pressure, or aldosterone levels. ${ }^{42}$ Unique to evacetrapib was a significant reduction in TG levels, especially at the $500 \mathrm{mg}$ daily dose. ${ }^{42}$

While anacetrapib and evacetrapib have provided encouraging clinical results, many questions still remain. To date, there have been no endpoint studies on anacetrapib or evacetrapib, although these are underway. Additionally, the integrated effects of these agents on HDL subclasses and composition, and especially on HDL functionality, are unclear.

\section{Dalcetrapib: pharmacology}

Among the CETP inhibitors, dalcetrapib appeared to have a distinctive mechanism of action and thus effect on clinical variables. Specifically, the increases in HDL cholesterol concentrations were much less pronounced with dalcetrapib (approximately 30\% with the $600 \mathrm{mg}$ dose) than with either anacetrapib or evacetrapib (approximately 120\%-130\% at full doses). Also, dalcetrapib had little effect on LDL cholesterol, in contrast to the LDL lowering of up to $40 \%$ seen with the other agents.

The earlier observation that torcetrapib treatment led to CE accumulation in HDL and selectively increased the level of spherical HDL particles ${ }^{43}$ provided a clue to the possible mechanistic differences between dalcetrapib and the other CETP inhibitors. Rather than the virtually complete inhibition of CETP's transfer function seen with the other agents, dalcetrapib is a weaker inhibitor. This supported the concept that dalcetrapib was a CETP modulator rather than a full CETP inhibitor. Further, dalcetrapib was postulated to induce a conformational change in CETP, allowing the transfer of $\mathrm{CE}$ from $\mathrm{HDL}_{3}$ to $\mathrm{HDL}_{2}$ and preserving the formation of pre- $\beta$ HDL particles. ${ }^{44}$ So while it raised HDL cholesterol considerably less than the other CETP inhibitors, ${ }^{45}$ the apparent selectivity of dalcetrapib for homotransfer of CE among HDL particles raised hope and expectations for a preferential antiatherogenic function. ${ }^{21}$ In contrast, the heterotransfer of CE between HDL and apo B-containing lipoproteins, which characterizes the other CETP inhibitors, could have explained why torcetrapib had no effect on atheroma and a negative effect on outcomes..$^{29,31}$ In animal studies, it appears that HDL particles that are formed secondarily to treatment with dalcetrapib, but not necessarily with anacetrapib, promote RCT and in vitro efflux of cholesterol from macrophages. ${ }^{44,46}$ These studies also showed differential effects of these agents on HDL subpopulations, as only anacetrapib inhibited transfer of $\mathrm{CE}$ from $\mathrm{HDL}_{3}$ to $\mathrm{HDL}_{2}$ and also formed pre- $\beta \mathrm{HDL}$ particles. ${ }^{44}$ Based on these findings, there was considerable optimism regarding dalcetrapib, and Roche undertook a major comprehensive program of clinical trials.

\section{Safety and tolerability of dalcetrapib}

After the failure of torcetrapib, concerns over a "class effect" on adverse outcomes led to stringent monitoring of the safety and efficacy of subsequent CETP-targeting agents. In early studies, dalcetrapib was found neither to cause hypertension in rats nor to increase expression of the renin-angiotensinaldosterone-system mRNA in the adrenal glands and aortas of rats. ${ }^{47}$ Importantly, the absence of any blood-pressure effects and aldosterone secretion was also seen in humans after 4 weeks. $^{48}$

Other adverse effects beyond the anticipated bloodpressure changes were also studied. Dalcetrapib had no effect on the cardiac QT interval in healthy subjects following multiple dosing regimens. ${ }^{49}$ No changes in mesenteric lymph nodes as measured by MRI were seen in patients on dalcetrapib. ${ }^{48}$ In the Phase I studies administering highdose dalcetrapib to healthy men, Derks et al reported no serious adverse events. ${ }^{50}$ In the four-week-long Phase II trial by Stein et al, the only adverse events of dalcetrapib compared to placebo were seen only in patients receiving high doses (900 mg), and they included only gastrointestinal symptoms. ${ }^{45}$ Dalcetrapib also has minimal interactions with other medications, including statins $\mathrm{s}^{35,51}$ and ezetimibe, ${ }^{52}$ and was even tolerated when coadministered with ketoconazole, a cytochrome P450 3A4 inhibitor. ${ }^{53}$

\section{Efficacy of dalcetrapib and comparative studies}

Early clinical studies of dalcetrapib demonstrated positive results on biochemical and surrogate outcomes. de Grooth et al showed, in 198 patients with mild hyperlipidemia, that dalcetrapib at doses from 300 to $900 \mathrm{mg}$ for 4 weeks decreased CETP activity by up to $37 \%$, increased HDL cholesterol by $34 \%$, decreased LDL cholesterol by $7 \%$, and had no effect on TG. ${ }^{55}$

Subsequent studies also showed biochemical efficacy. For instance, Kuivenhoven et $\mathrm{al}^{54}$ and Hermann et $\mathrm{al}^{41}$ showed a $28 \%$ and $26 \%$ respective increase in HDL cholesterol with the $600 \mathrm{mg}$ daily dose. Hermann et al also demonstrated a fall in TG by $22 \%$, as well as an increase in brachial flowmediated dilation (FMD), a presumed representation of endothelial function, when patients with low baseline HDL cholesterol were on dalcetrapib treatment. However, no changes to FMD or to serum markers of inflammation were seen with dalcetrapib treatment, overall. ${ }^{41}$ Finally, Stein et al 
conducted Phase II trials evaluating efficacy at 4 weeks and 12 weeks. ${ }^{45}$ These demonstrated that dalcetrapib treatment increased HDL cholesterol by up to $36 \%$, setting the stage for the large clinical trials.

\section{The dal-HEART program}

The optimism for and commitment to dalcetrapib were underscored by embarking on the Dalcetrapib HDL Evaluation, Atherosclerosis and Reverse Cholesterol Transport (dal-HEART) program, which was comprised of a series of double-blind, randomized, placebo-controlled studies assessing hard clinical outcomes and some imaging markers. The dal-HEART program included five principal studies, specifically dal-PLAQUE,${ }^{56}$ dal-VESSEL,${ }^{57}$ dal-PLAQUE $2,{ }^{58}$ dal-ACUTE, ${ }^{59}$ and dal-OUTCOMES. ${ }^{60}$

Dal-PLAQUE ${ }^{56}$ was a Phase IIB double-blind, randomized, placebo-controlled study, which showed that dalcetrapib treatment reduced the increase in vessel plaque area in patients already on statin therapy. The study assessed the effect of dalcetrapib (600 mg daily) versus placebo on atherosclerotic plaque in 130 subjects with high coronary artery disease risk. In addition, the presence of vessel wall thickening was measured by positron emission tomography/ computed tomography (PET/CT) and magnetic resonance imaging (MRI). One hundred and thirty patients received either dalcetrapib or placebo for up to 2 years. Eighty-six percent of the subjects were taking statins and had an LDL cholesterol level of less than $2.6 \mathrm{mmol} / \mathrm{L}$. Primary endpoints were assessed by MRI of plaque size and PET/CT scanning of target-plaque-to-background-blood ratio. The authors observed a significant difference $(P<0.04)$ in the progression of plaque in the dalcetrapib group compared to the placebo group as assessed by MRI. Although a 7\% reduction in the most diseased carotid segment was seen in the dalcetrapib group, no significant difference $(P=0.08)$ in the wall thickness of the right and left carotid arteries or the ascending thoracic aorta was found when measured by PET/CT.

Overall, dal-PLAQUE found that dalcetrapib was safe and possibly led to regression of carotid lesions and reduction of vessel inflammation. ${ }^{56}$ These mild changes observed must be interpreted in context, as the patients were concurrently on effective statin therapy, illustrating that the plaque benefit was incremental to that of statins. ${ }^{17}$ Interestingly, these benefits correlated with the degree of HDL cholesterol increase. The dalcetrapib group also showed a 7\% reduction in LDL cholesterol beyond that accounted for by their statin treatment. Although not powered statistically to look at CVD events, in dal-PLAQUE there were fewer CVD events in the dalcetrapib group than in the placebo group (3\% vs $11 \%$, respectively).

In the recent dal-VESSEL trial, ${ }^{57}$ patients with CVD or equivalent and low HDL cholesterol were treated with dalcetrapib $600 \mathrm{mg}$ or placebo for 36 weeks. Here, the primary endpoints evaluated were the change in brachial FMD after 12 and 36 weeks. Similar to prior studies, dalcetrapib treatment led to a 50\% reduction in CETP activity and a $31 \%$ increase in HDL cholesterol. Levels of apo A-I were also increased, but there was no change to LDL cholesterol. Despite these favorable biochemical changes, no significant differences were observed for FMD at any point of the study. Ambulatory 24-hour systolic and diastolic blood pressures at 4, 12, and 36 weeks were not altered in the dalcetrapib arm, and there were no increases in nitric oxide-dependent endothelial function or markers of oxidative stress and inflammation. ${ }^{57}$

Unpublished studies in the dalcetrapib clinical program include dal-PLAQUE2, dal-ACUTE, and dal-OUTCOMES. Dal-PLAQUE2 was designed to evaluate dalcetrapib's effect on atherosclerosis by measuring coronary plaque in 900 patients by intravascular carotid B-mode ultrasound imaging and coronary angioplasty. ${ }^{61}$ Dal-ACUTE (NCT01323153) evaluated the use of dalcetrapib in 300 patients after acute coronary syndrome. ${ }^{59}$ The largest study, namely dal-OUTCOMES, was a multicenter, randomized, doubleblinded, placebo-controlled Phase III trial ${ }^{60}$ that randomized 15,600 patients to either dalcetrapib or placebo 4 to 12 weeks after acute coronary syndrome. Patients were monitored for major cardiovascular events, including coronary artery disease death, acute MI, unstable angina requiring hospital admission, cardiac arrest, or stroke. In May 2012, Roche announced that it was terminating the dal-OUTCOMES trial 2 years early due to its lack of efficacy, and was simultaneously terminating the entire dalcetrapib program.

\section{Implications of termination of the dalcetrapib development program}

The CVD prevention community has been left somewhat shell-shocked after the recent turn of events related to dalcetrapib, which, to say the least, were unexpected and surprising. Dalcetrapib lacked the known off-target effects of torcetrapib. Further, dalcetrapib's contribution to the reduction of atherosclerotic progression and vascular inflammation without adversely affecting blood pressure or aldosterone ${ }^{56}$ seemed encouraging. But despite these encouraging safety and efficacy signals from the early-phase clinical trials, and the evidence favoring the unique mechanism of CETP modulation, the Phase III dal-OUTCOMES trial 
was terminated prematurely. ${ }^{62}$ Full details surrounding this termination should emerge soon, and hopefully there will be full publication of them. However, the termination appears to have been due to lack of efficacy rather than to increased risk of harm or deleterious effects. ${ }^{63}$

Considering the prior failures of ILLUMINATE and AIM-HIGH, dal-OUTCOMES adds further evidence that targeting HDL does not improve CVD risk. But is the HDL hypothesis wrong? Or does the way in which HDL is raised matter? Perhaps CETP inhibition is not the best way to raise HDL. Further evidence that HDL itself may simply be a biomarker rather than a causal factor for CVD came from recent genetic studies performed in tens of thousands of patients, which found (1) an inverse association of CHD risk in patients with genetically reduced CETP function ${ }^{64}$ and (2) that certain genetic variants that raise HDL over a lifetime do not lower risk of myocardial infarction. ${ }^{65}$ Although these results seem to further undermine the importance of HDL, it may be too soon to bury HDL as a target for treatment.

Certainly, serum HDL cholesterol level is an exemplary biomarker and risk predictor. But that does not automatically make it a therapeutic target. We are beginning to appreciate that it is perhaps the quality and function of HDL, or more likely of a specific HDL subfraction, that may be mechanistically more important in causing CVD. In other words, not all HDL particles are equally protective, and current clinical assays that simply measure the total quantity of HDL cholesterol cannot assess these qualitative functional differences. It remains unclear which, if any, of HDL's many roles give protection from cardiovascular effects, since its anti-inflammatory, antioxidant, antithrombotic, ${ }^{13}$ and other functional properties are still under investigation.

Epidemiological studies have shown that low HDL does confer an increased risk of CVD, ${ }^{3}$ and there have been reports that pharmacologic CETP inhibition does not impair the lipid-mobilizing activities of HDL. ${ }^{66}$ However, pharmacologically raising HDL has yet to show benefits in those at residual risk of CVD. It has not yet been proven that there is a causal relationship between HDL cholesterol level and cardioprotection, and low HDL cholesterol may be simply a marker of other disease processes.

Although the "HDL hypothesis" has been weakened lately, it is worth noting that large-scale outcome studies of anacetrapib and evacetrapib are ongoing and have not been terminated. Furthermore, other types of CETP inhibitors are under development. ${ }^{67}$ To date, the effects of anacetrapib and evacetrapib on the progression of atherosclerosis or CVD endpoints are unknown, but both profoundly increase HDL cholesterol, compared to dalcetrapib. Perhaps as important, anacetrapib and evacetrapib also reduce LDL cholesterol by $35 \%-40 \%$. So it is a distinct possibility that CVD outcome trials with anacetrapib and evacetrapib added to statins will show a benefit, but this may not have anything to do with the effect on HDL. The experience with dalcetrapib provides few clues to help predict the outcomes of trials with the remaining CETP inhibitors. These are important studies that might finally settle the debate over whether either HDL raising or CETP inhibition are useful approaches to CVD risk reduction.

\section{Disclosure}

Dr Hegele is a Career Investigator of the Heart and Stroke Foundation of Ontario, holds the Edith Schulich Vinet Canada Research Chair (Tier I) in Human Genetics, the Martha G Blackburn Chair in Cardiovascular Research, and the Jacob J Wolfe Distinguished Medical Research Chair at the University of Western Ontario. This work was supported by CIHR (MOP13430, MOP-79523, CTP-79853), and by the Heart and Stroke Foundation of Ontario (NA-6059, T-6018, PRG-4854). The authors report no other conflicts of interest in this work.

\section{References}

1. Baigent C, Keech A, Kearney P, Al E. Efficacy and safety of cholesterol-lowering treatment: prospective meta-analysis of data from 90,056 participants in 14 randomised trials of statins. Lancet. 2005;366(9493):1267-1278.

2. Gordon T, Castelli WP, Hjortland MC, Kannel WB, Dawber TR. High density lipoprotein as a protective factor against coronary heart disease. The Framingham study. Am J Med. 1977;62(5):707-714.

3. Gordon DJ, Probstfield JL, Garrison RJ, et al. High-density lipoprotein cholesterol and cardiovascular disease. Four prospective American studies. Circulation. 1989;79(1):8-15.

4. Joy T, Hegele RA. Is raising HDL a futile strategy for atheroprotection? Nat Rev Drug Discov. 2008;7(2):143-155.

5. Asztalos BF, Roheim PS, Milani RL, et al. Distribution of ApoA-Icontaining HDL subpopulations in patients with coronary heart disease. Arterioscler Thromb Vasc Biol. 2000;20(12):2670-2676.

6. Rye K-A, Bursill CA, Lambert G, Tabet F, Barter PJ. The metabolism and anti-atherogenic properties of HDL. J Lipid Res. 2009; 50 Suppl:S195-S200.

7. Tabet F, Rye K-A. High-density lipoproteins, inflammation and oxidative stress. Clin Sci. 2009;116(2):87-98.

8. Johns DG, Duffy J, Fisher T, Hubbard BK, Forrest MJ. On- and offtarget pharmacology of torcetrapib: current understanding and implications for the structure activity relationships (SAR), discovery and development of cholesteryl ester-transfer protein (CETP) inhibitors. Drugs. 2012;72(4):491-507.

9. Matsuura F. HDL from CETP-deficient subjects shows enhanced ability to promote cholesterol efflux from macrophages in an apoE- and ABCG1-dependent pathway. J Clin Invest. 2006;116(5):1435-1442.

10. Asztalos BF, Collins D, Horvath KV, Bloomfield HE, Robins SJ, Schaefer EJ. Relation of gemfibrozil treatment and high-density lipoprotein subpopulation profile with cardiovascular events in the Veterans Affairs High-Density Lipoprotein Intervention Trial. Metab Clin Exp. 2008;57(1):77-83. 
11. Ballantyne FC, Clark RS, Simpson HS, Ballantyne D. High density and low density lipoprotein subfractions in survivors of myocardial infarction and in control subjects. Metab Clin Exp. 1982;31(5):433-437.

12. Patsch JR, Karlin JB, Scott LW, Smith LC, Gotto AM. Inverse relationship between blood levels of high density lipoprotein subfraction 2 and magnitude of postprandial lipemia. Proc Natl Acad Sci U S A. 1983;80(5):1449-1453.

13. Cheung V, Hegele RA. CETP Inhibitors: will they live up to their promise? Curr Cardiovasc Risk Rep. 2011;6(1):4-11.

14. Asztalos BF, Collins D, Cupples LA, et al. Value of high-density lipoprotein (HDL) subpopulations in predicting recurrent cardiovascular events in the Veterans Affairs HDL Intervention Trial. Arterioscler Thromb Vasc Biol. 2005;25(10):2185-2191.

15. Asztalos BF, Cupples LA, Demissie S, et al. High-density lipoprotein subpopulation profile and coronary heart disease prevalence in male participants of the Framingham Offspring Study. Arterioscler Thromb Vasc Biol. 2004;24(11):2181-2187.

16. Khera AV, Cuchel M, la Llera-Moya M de, et al. Cholesterol efflux capacity, high-density lipoprotein function, and atherosclerosis. $N$ Engl J Med. 2011;364(2):127-135.

17. Doggrell SA. Dalcetrapib - restoring belief in modulating CETP as a beneficial mechanism in cardiovascular disease. Expert Opin Investig Drugs. 2012;21(4):569-573.

18. AIM-HIGH Investigators, Boden WE, Probstfield JL, et al. Niacin in patients with low HDL cholesterol levels receiving intensive statin therapy. N Engl J Med. 2011;365(24):2255-2267.

19. Gurfinkel R, Joy TR. Anacetrapib: hope for CETP inhibitors? Cardiovasc Ther. 2011;29(5):327-339.

20. HPS2-THRIVE [webpage on the Internet]. Oxford: Clinical Trial Service Unit [updated June 14, 2012]. Available from: http://www. ctsu.ox.ac.uk/ thrive/. Accessed June 18, 2011.

21. Niesor EJ. Different effects of compounds decreasing cholesteryl ester transfer protein activity on lipoprotein metabolism. Curr Opin Lipidol. 2011;22(4):288-295.

22. Rader DJ. Molecular regulation of HDL metabolism and function: implications for novel therapies. J Clin Invest. 2006;116(12):3090-3100.

23. Morehouse LA, Sugarman ED, Bourassa PA, et al. Inhibition of CETP activity by torcetrapib reduces susceptibility to diet-induced atherosclerosis in New Zealand White rabbits. J Lipid Res. 2007;48(6): 1263-1272.

24. Clark RW, Sutfin TA, Ruggeri RB, et al. Raising high-density lipoprotein in humans through inhibition of cholesteryl ester transfer protein: an initial multidose study of torcetrapib. Arterioscler Thromb Vasc Biol. 2004;24(3):490-497.

25. McKenney JM, Davidson MH, Shear CL, Revkin JH. Efficacy and safety of torcetrapib, a novel cholesteryl ester transfer protein inhibitor, in individuals with below-average high-density lipoproteincholesterol levels on a background of at orvastatin. J Am Coll Cardiol. 2006;48(9):1782-1790.

26. Tall AR, Yvan-Charvet L, Wang N. The failure of torcetrapib: was it the molecule or the mechanism? Arterioscler Thromb Vasc Biol. 2007;27(2):257-260.

27. Mullard A. Learning lessons from Pfizer's $\$ 800$ million failure. Nat Rev Drug Discov. 2011;10(3):163-164.

28. Barter PJ, Caulfield M, Eriksson M, et al. Effects of torcetrapib in patients at high risk for coronary events. N Engl J Med. 2007;357(21): 2109-2122.

29. Nissen SE, Tardif J-C, Nicholls SJ, et al. Effect of torcetrapib on the progression of coronary atherosclerosis. N Engl J Med. 2007;356(13): 1304-1316.

30. Kastelein JJP, van Leuven SI, Burgess L, et al. Effect of torcetrapib on carotid atherosclerosis in familial hypercholesterolemia. $N$ Engl J Med. 2007;356(16):1620-1630.

31. Bots ML, Visseren FL, Evans GW, et al. Torcetrapib and carotid intima-media thickness in mixed dyslipidaemia (RADIANCE 2 study): a randomised, double-blind trial. Lancet. 2007;370(9582): $153-160$.
32. Nicholls SJ, Tuzcu EM, Brennan DM, Tardif J-C, Nissen SE. Cholesteryl ester transfer protein inhibition, high-density lipoprotein raising, and progression of coronary atherosclerosis: insights from ILLUSTRATE (Investigation of Lipid Level Management Using Coronary Ultrasound to Assess Reduction of Atherosclerosis by CETP Inhibition and HDL Elevation). Circulation. 2008;118(24):2506-2514.

33. Hu X, Dietz JD, Xia C, et al. Torcetrapib induces aldosterone and cortisol production by an intracellular calcium-mediated mechanism independently of cholesteryl ester transfer protein inhibition. Endocrinology. 2009;150(5):2211-2219.

34. Forrest MJ, Bloomfield D, Briscoe RJ, et al. Torcetrapib-induced blood pressure elevation is independent of CETP inhibition and is accompanied by increased circulating levels of aldosterone. Br J Pharmacol. 2008;154(7):1465-1473.

35. Miyares MA. Anacetrapib and dalcetrapib: two novel cholesteryl ester transfer protein inhibitors. Ann Pharmacother. 2011;45(1):84-94.

36. Cannon CP, Shah S, Dansky HM, et al. Safety of anacetrapib in patients with or at high risk for coronary heart disease. $N$ Engl J Med. 2010;363(25):2406-2415.

37. Bloomfield D, Carlson GL, Sapre A, et al. Efficacy and safety of the cholesteryl ester transfer protein inhibitor anacetrapib as monotherapy and coadministered with atorvastatin in dyslipidemic patients. Am Heart J. 2009;157(2):352-360. e2.

38. Krishna R, Garg A, Jin B, et al. Assessment of a pharmacokinetic and pharmacodynamic interaction between simvastatin and anacetrapib, a potent cholesteryl ester transfer protein (CETP) inhibitor, in healthy subjects. Br J Clin Pharmacol. 2009;67(5):520-526.

39. Krishna R, Anderson MS, Bergman AJ, et al. Effect of the cholesteryl ester transfer protein inhibitor, anacetrapib, on lipoproteins in patients with dyslipidaemia and on 24-h ambulatory blood pressure in healthy individuals: two double-blind, randomised placebo-controlled phase I studies. Lancet. 2007;370(9603):1907-1914.

40. Krishna R, Bergman AJ, Jin B, et al. Multiple-dose pharmacodynamics and pharmacokinetics of anacetrapib, a potent cholesteryl ester transfer protein (CETP) inhibitor, in healthy subjects. Clin Pharmacol Ther. 2008;84(6):679-683.

41. Hermann F, Enseleit F, Spieker LE, et al. Cholesterylestertransfer protein inhibition and endothelial function in type II hyperlipidemia. Thromb Res. 2009;123(3):460-465.

42. Nicholls SJ, Brewer HB, Kastelein JJ, et al. Effects of the CETP inhibitor evacetrapib administered as monotherapy or in combination with statins on HDL and LDL cholesterol: a randomized controlled trial. JAMA. 2011;306(19):2099-2109.

43. Brousseau ME, Schaefer EJ, Wolfe ML, et al. Effects of an inhibitor of cholesteryl ester transfer protein on HDL cholesterol. N Engl J Med. 2004;350(15):1505-1515.

44. Niesor EJ, Magg C, Ogawa N, et al. Modulating cholesteryl ester transfer protein activity maintains efficient pre- $\beta$-HDL formation and increases reverse cholesterol transport. J Lipid Res. 2010;51(12): 3443-3454

45. Stein EA, Stroes ESG, Steiner G, et al. Safety and tolerability of dalcetrapib. Am J Cardiol. 2009;104(1):82-91.

46. Kobayashi J, Okamoto H, Otabe M, Bujo H, Saito Y. Effect of HDL, from Japanese white rabbit administered a new cholesteryl ester transfer protein inhibitor JTT-705, on cholesteryl ester accumulation induced by acetylated low density lipoprotein in $\mathrm{J} 774$ macrophage. Atherosclerosis. 2002;162(1):131-135.

47. Stroes E, Kastelein J, Bénardeau A, et al. Dalcetrapib: no off-target toxicity on blood pressure or on genes related to the renin-angiotensin-aldosterone system in rats. Br J Pharmacol. 2009;158(7): 1763-1770

48. Stein EA, Roth EM, Rhyne JM, Burgess T, Kallend D, Robinson JG. Safety and tolerability of dalcetrapib (RO4607381/JTT-705): results from a 48-week trial. Eur Heart J. 2010;31(4):480-488.

49. Derks M, Abt M, Mwangi A, Meneses-Lorente G. Lack of effect of dalcetrapib on QT interval in healthy subjects following multiple dosing. Eur J Clin Pharmacol. 2010;66(8):775-783. 
50. Derks M, Anzures-Cabrera J, Turnbull L, Phelan M. Safety, tolerability and pharmacokinetics of dalcetrapib following single and multiple ascending doses in healthy subjects: a randomized, double-blind, placebo-controlled, Phase I study. 2011;31(5):325-335.

51. Derks M, Abt M, Phelan M, et al. Coadministration of dalcetrapib with pravastatin, rosuvastatin, or simvastatin: no clinically relevant drug-drug interactions. J Clin Pharmacol. 2010;50(10):1188-1201.

52. Derks M, Abt M, Phelan M. Lack of clinically relevant drug-drug interactions when dalcetrapib is co-administered with ezetimibe. $\mathrm{BrJ}$ Clin Pharmacol. 2010;70(6):825-833.

53. Derks M, Fowler S, Kuhlmann O. A single-center, open-label, onesequence study of dalcetrapib coadministered with ketoconazole, and an in vitro study of the S-methyl metabolite of dalcetrapib. Clin Ther. 2009;31(3):586-599.

54. de Grooth GJ, Kuivenhoven JA, Stalenhoef AF, et al. Efficacy and safety of a novel cholesteryl ester transfer protein inhibitor, JTT-705, in humans: a randomized phase II dose-response study. Circulation. 2002;105(18):2159-2165.

55. Kuivenhoven JA, de Grooth GJ, Kawamura H, et al. Effectiveness of inhibition of cholesteryl ester transfer protein by JTT-705 in combination with pravastatin in type II dyslipidemia. Am J Cardiol. 2005;95(9):1085-1088.

56. Fayad ZA, Mani V, Woodward M, et al. Safety and efficacy of dalcetrapib on atherosclerotic disease using novel non-invasive multimodality imaging (dal-PLAQUE): a randomised clinical trial. Lancet. 2011; 378(9802):1547-1559.

57. Luscher TF, Taddei S, Kaski JC, et al. Vascular effects and safety of dalcetrapib in patients with or at risk of coronary heart disease: the dal-VESSEL randomized clinical trial. Eur Heart J. 2012;33(7):857-865.

58. Roche. A study of the effect of dalcetrapib on artherosclerotic disease in patients with coronary artery disease. In: ClinicalTrials.gov [website on the Internet]. Bethesda, MD: US National Library of Medicine; 2012 [updated August 15, 2012]. Available from: http://clinicaltrials.gov/ ct2/show/NCT01059682. NLM identifier: NCT01059682. Accessed September 9, 2012.
59. Roche. A study of dalcetrapib in patients hospitalized for an acute coronary syndrome (Dal-ACUTE) In: ClinicalTrials.gov [website on the Internet]. Bethesda, MD: US National Library of Medicine; 2012 [updated July 5, 2012]. Available from: http://clinicaltrials.gov/ ct2/show/NCT01323153. NLM identifier: NCT01323153. Accessed September 3, 2012.

60. Schwartz GG, Olsson AG, Ballantyne CM, et al. Rationale and design of the dal-OUTCOMES trial: efficacy and safety of dalcetrapib in patients with recent acute coronary syndrome. Am Heart J. 2009;158(6): 896-901. e3.

61. Robinson JG. Dalcetrapib: a review of Phase II data. Expert Opin Investig Drugs. 2010;19(6):795-805.

62. F Hoffmann-La Roche Ltd. Roche provides update on Phase III study of dalcetrapib [media release]. Basel: F Hoffmann-La Roche Ltd; 2012 [May 7]. Available from: http://www.roche.com/media/media_releases/ med-cor-2012-05-07.htm. Accessed. June 11, 2012.

63. Miller R. Roche stops dalcetrapib trial for lack of benefit. Montreal: theheart.org; 2012. Available from: http://www.theheart.org/article/1395141.do. Accessed May 30, 2012.

64. Thompson A, Di Angelantonio E, Sarwar N, et al. Association of cholesteryl ester transfer protein genotypes with CETP mass and activity, lipid levels, and coronary risk. JAMA. 2008;299(23):2777-2788.

65. Voight BF, Peloso GM, Orho-Melander M, et al. Plasma HDL cholesterol and risk of myocardial infarction: a mendelian randomisation study. Lancet. 2012;380(9841):572-580.

66. Nicholls SJ. Evacetrapib. Curr Cardiol Rep. 2012;14(3):245-250.

67. Harikrishnan LS, Finlay HJ, Qiao JX, et al. Diphenylpyridylethanamine (DPPE) derivatives as cholesteryl ester transfer protein (CETP) Inhibitors. J Med Chem. 2012;55(13):6162-6175.
Drug Design, Development and Therapy

\section{Publish your work in this journal}

Drug Design, Development and Therapy is an international, peerreviewed open-access journal that spans the spectrum of drug design and development through to clinical applications. Clinical outcomes, patient safety, and programs for the development and effective, safe, and sustained use of medicines are a feature of the journal, which

\section{Dovepress}

has also been accepted for indexing on PubMed Central. The manuscript management system is completely online and includes a very quick and fair peer-review system, which is all easy to use. Visit http://www.dovepress.com/testimonials.php to read real quotes from published authors. 